\title{
Open Surgery for Ingested Gastric Foreign Bodies in the Era of Endoscopy and Minimally Invasive Surgery
}

\author{
Vijay Waman Dhakre, M.S., FHPB, FMAS ${ }^{1 *}$, Aparna Muraleedharan Warrier², Chetan Rathod, \\ MS $^{3}$, Kayomarz S. Sethna, MS FRCS ${ }^{4}$

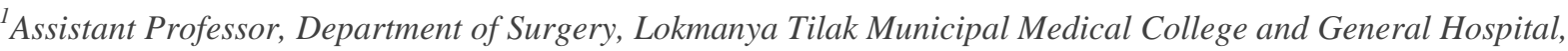 \\ Sion, Mumbai, Maharashtra India \\ ${ }^{2}$ Surgical Resident, Department of Surgery, Lokmanya Tilak Municipal Medical College and General Hospital, \\ Sion, Mumbai, Maharashtra India \\ ${ }^{3}$ Associate Professor, Department of Surgery, Lokmanya Tilak Municipal Medical College and General Hospital, \\ Sion, Mumbai, Maharashtra India \\ ${ }^{4}$ Professor of Surgery, Department of Surgery, Lokmanya Tilak Municipal Medical College and General Hospital,
} Sion, Mumbai, Maharashtra India

*Corresponding Author: Dr Vijay Dhakre, $1^{\text {st }}$ Floor Department of General Surgery, Lokmanya Tilak Municipal Medical College and General hospital, Dr BR Ambedkar Road, Sion, Mumbai - 400022, India. Email:vddrvijayd@gmail.com

\begin{abstract}
29-year-old gentleman presented with a large sharp knife ingestion and had an acute sharp pain in abdomen. Open surgical approach was adopted successfully due to high suspicion of visceral injury and to avoid foreign body removal injuries. Large-sharp object like knife is considered for emergency intervention. Specialized skills \& equipment are needed for endoscopic approach; but open surgical approach can be safely used if these are not available. Also, one of the major aims of approach should be to minimize retrieval injury.
\end{abstract}

Keywords: Large-sharp knife, foreign body, Endoscopy, Retrieval injury.

\section{DESCRIPTION}

29-year-old gentleman non-compliant for the treatment of schizophrenia started a year ago presented with ingestion of a knife 4 hours ago. He was hemodynamically stable and his abdomen was non-tender and non-distended.

X-ray of the Chest and abdomen showed a long foreign body along the vertical axis of the stomach with its sharp blade placed caudally across the Gastro-oesophageal junction (GEJ). [Figure: 1] Upper GI endoscopy was planned for him but he started experiencing sudden sharp pain in the upper abdomen without any clinical signs of peritonitis.
$\mathrm{X}$-ray of the abdomen was repeated immediately to rule out any visceral perforation. It was noted that the whole knife has migrated lower down and crossed the GEJ and is now placed with blade vertically downwards more caudal in the upper abdomen. [Figure: 2] This changed scenario arouses suspicion of gastric injury and prompted us to take up an exploratory laparotomy approach. Laparotomy showed an intact anterior and posterior stomach wall and a normal GEJ. The anterior gastrotomy was done in the antrum and the intact knife was delivered. [Figure: 3] Gastrotomy closed in the standard technique. The patient was discharged on a postoperative day 3 without any complications. 


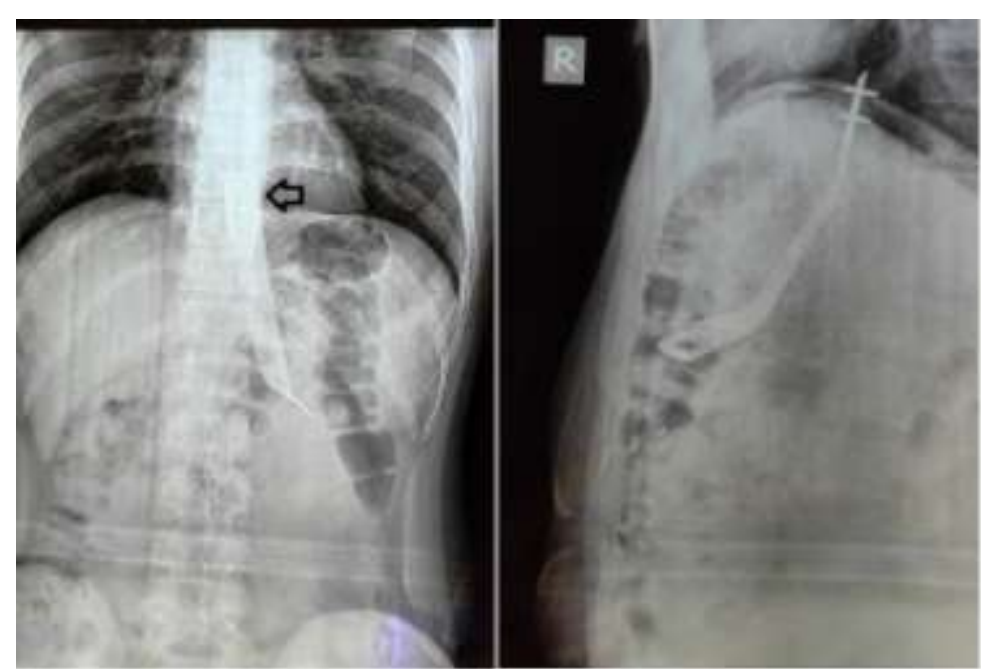

Figure1. Plain X-ray of chest and abdomen of patient at presentation. Note the position of the knife (arrow) suggesting location across GEJ

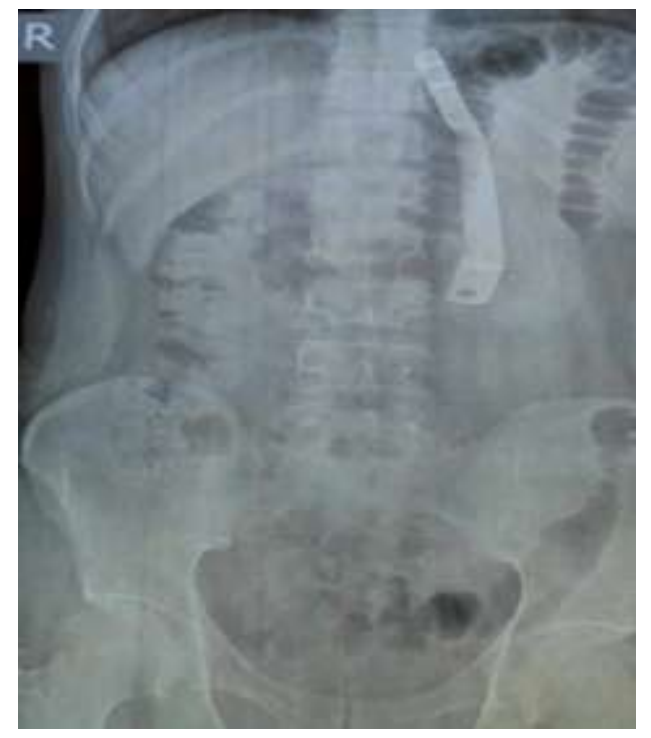

Figure2. X-ray Abdomen showing migration of the knife caudally as compared to position at presentation

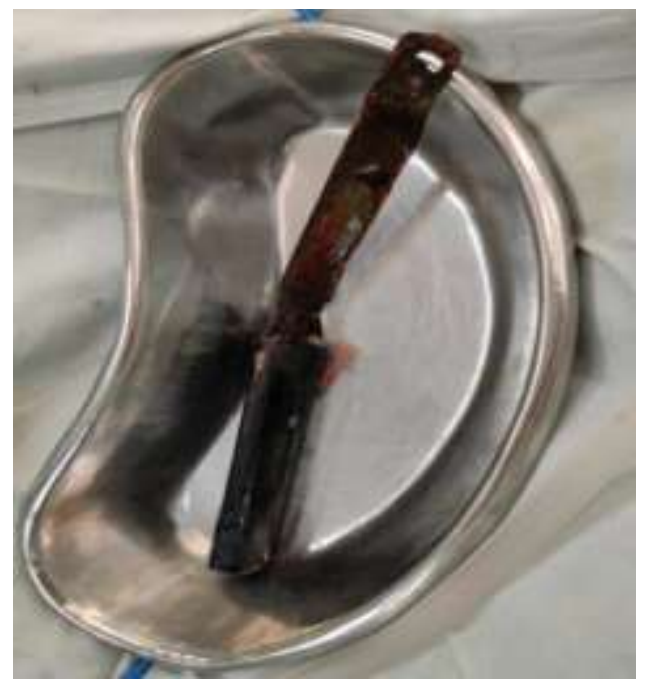

Figure3. Knife retrieved after Gastrotomy 
The majority of the foreign bodies can be retrieved via the endoscopic route (10\%-20\%), but surgical procedures are needed in around $1 \%$ of patients and are kept reserved for endoscopic failure and visceral perforation. [1]

Injuries to the nasopharynx, oesophageal mucosa, GEJ, and stomach is the most common issue and should be addressed by the endoscopist and surgeon. One of the aims should be to protect the airway either by elective endotracheal intubation and use of a rigid esophagoscope. [2] Removal from oesophagus can be tricky as there are not only chances of injuring oesophagus and upper GI tract but also thoracic viscera. The range of retrieval injury can range from $15 \%-35 \%$ and is more in less experienced hands. [3]

To avoid such injuries various techniques (viz. dual snare technique) and a combination of 2 or more special instruments are now routinely in use like rat-tooth or alligator forceps, snares used for polypectomies or Dormier baskets, protective hoods to avoid mucosal injury once the sharp object is grasped. [2,4] Use of over-tubes (up to $45 \mathrm{~cm}$ long) is recommended to retrieve sharp and long foreign body like blades and knives to avoid injury to the stomach, GEJ, oesophagus, and oropharynx. [1]

Open surgery is favoured in situations where there is slight suspicion of visceral perforation or peritonitis as it also gives us access to inspect the viscera, give lavages and repair the injury. It is often the preferred approach in cases where the foreign body has migrated out of the GI tract. [5] Open surgery can be done in centres where such advanced endoscopies or expertise are not available. In our case patient had ingested a long sharp knife and which had migrated lower in the stomach causing sudden sharp pain which prompted us to use the open surgery approach safely.

\section{Conclusions}

- Open surgery should be planned in cases of larger and sharper foreign body specially in institutions with low expertise, lack of specific instruments and or expertise.

- Approach to removal of such large-sharp objects should be strategically tailored by a multi-disciplinary team to reduce iatrogenic injuries.

- For multiple sharp foreign bodies combination approaches like open, laparoscopic, thoracoscopic and endoscopic should be used to minimize damage during retrieval.

\section{REFERENCES}

[1] Birk M, Bauerfeind P, Deprez PH, et al. Removal of foreign bodies in the upper gastrointestinal tract in adults: European Society of Gastrointestinal Endoscopy (ESGE) Clinical Guideline. Endoscopy. 2016;48(5):489-496. doi:10.1055/s-0042-100456

[2] ASGE Standards of Practice Committee, Ikenberry SO, Jue TL, et al. Management of ingested foreign bodies and food impactions. GastrointestEndosc. 2011;73(6):1085-1091. doi:10.1016/j.gie.2010.11.010

[3] Harvey E, Lee D, Giliberto JP. Real steak knives of cincinnati: Repeated foreign body ingestion with novel endoscopic removal. Laryngoscope. 2019;129(3):567-568. doi:10.1002/lary.27329

[4] Judge J, Le A, Wecowski J. Getting a Better Grip: A Novel Modification to the Dual Snare Technique for Removing Long Esophageal Foreign Bodies. Am Surg. 2020;86(5):541-543. doi: $10.1177 / 0003134820919728$

[5] Guo C, Yuan Y, Liu L. A Broken Fruit Knife: Half in the Bronchus and Half in the Duodenum. Indian J Surg. 2017;79(1):75-76. doi:10.1007/s12262-017-1600-8.

Citation: Vijay Waman Dhakre, Aparna Muraleedharan Warrier, Chetan Rathod, Kayomarz S. Sethna. "Open Surgery for Ingested Gastric Foreign Bodies in the Era of Endoscopy and Minimally Invasive Surgery". ARC Journal of Clinical Case Reports. 2021; 7(1):4-6. DOI: https://doi.org/10.20431/2455-9806.0701002.

Copyright: () 2021 Authors. This is an open-access article distributed under the terms of the Creative Commons Attribution License, which permits unrestricted use, distribution, and reproduction in any medium, provided the original author and source are credited. 